\title{
Non-LTE diagnostics of velocity fields during the gradual phase of a solar flare
}

\author{
A. Berlicki ${ }^{1,2}$, P. Heinzel ${ }^{3}$, B. Schmieder ${ }^{1,4}$, P. Mein $^{1}$, and N. Mein ${ }^{1}$ \\ 1 Observatoire de Paris, Section de Meudon, LESIA, 92195 Meudon Principal Cedex, France \\ e-mail: arkadiusz.berlicki@obspm. fr \\ 2 Astronomical Institute of the Wrocław University, ul. Kopernika 11, 51-622 Wrocław, Poland \\ 3 Astronomical Institute, Academy of Sciences of the Czech Republic, 25165 Ondřejov, Czech Republic \\ 4 Institute of Theoretical Astrophysics, University of Oslo, Blindern, 0315 Oslo, Norway
}

Received 14 May 2004 / Accepted 13 September 2004

\begin{abstract}
We perform an analysis of the velocity field within the $\mathrm{H} \alpha$ ribbons during the gradual phase of an $\mathrm{M} 1.0$ solar flare observed on October 22, 2002. We use spectroscopic observations performed with the German VTT (Vacuum Tower Telescope) working in the MSDP (Multichannel Subtractive Double Pass spectrograph) observing mode. From these observations the $\mathrm{H} \alpha$ line profiles in chosen areas of the flare ribbons were reconstructed and these observational profiles were compared with a grid of synthetic $\mathrm{H} \alpha$ line profiles calculated by the non-LTE radiative-transfer code. This code allows us to calculate different models of the chromosphere with a prescribed velocity field. By optimising the best fit between the observed and synthetic profiles we find the most appropriate models of the chromosphere and vertical structure of the velocity field in the analysed areas of the flare ribbons. By means of the non-LTE radiative-transfer calculations we show that in most analysed areas of the $\mathrm{H} \alpha$ flare ribbons the chromospheric plasma exhibited upward motion with a mean velocity of a few $\mathrm{km} \mathrm{s}^{-1}$. These results are consistent with previous estimates and support the scenario of a gentle evaporation during the gradual phase.
\end{abstract}

Key words. Sun: flares - Sun: chromosphere - line: profiles

\section{Introduction}

Various asymmetrical profiles of different spectral lines emitted from solar flares have been frequently observed. These asymmetries or line shifts observed both in X-rays as well as in chromospheric lines are caused by predominantly vertical mass motions in flaring layers. It is widely accepted that during chromospheric flares the plasma is evaporated into the corona providing material for loop prominences often observed as the so-called post-flare loops (Kopp 1976; Antiochos \& Sturrock 1978). Such evaporation should produce blue-shifted line profiles for the flares located close to the disk centre. For the hot plasma at temperatures of several MK this blue-shifted component, observed mainly during the impulsive phase, was detected in the X-ray spectra of many flares observed by the Yohkoh/BCS instrument (Doschek et al. 1994; Wülser et al. 1994; Schmieder et al. 1998; Berlicki et al. 2002). Plasma velocities deduced from Doppler-shift analysis are of the order of a few hundred $\mathrm{km} \mathrm{s}^{-1}$. This explosive evaporation is considered to be driven mainly by non-thermal electrons accelerated during the primary energy release (Antonucci et al. 1984) and may occur when the heating rate due to collisions of non-thermal electrons with the ambient plasma in upper layers of the chromosphere exceeds the chromospheric radiative losses (Fisher 1987). This occurs when the temperature of the heated region is above $2 \times 10^{5} \mathrm{~K}$ and thus this kind of evaporation cannot be seen in the chromospheric spectral lines of hydrogen or calcium which are formed in cooler plasma. Another mechanism which can drive the evaporation during the impulsive phase of flares is that of thermal conduction, however the role of this mechanism is still debated. Ji et al. (2004) found that both thermal conduction and non-thermal electrons play a role in the heating of the lower atmosphere even for the same flare kernel sites.

If a region of the chromosphere heated by non-thermal electrons is thick enough, then the rapid temperature increase produces an enhanced pressure in the heated region. This overpressure, besides the evaporation, also drives downwardmoving cool and dense chromospheric condensations (Fisher et al. 1985b) which seem to be responsible for red-shifts of the $\mathrm{H} \alpha$ line profiles reported by many authors (Švestka 1976; Ichimoto \& Kurokawa 1984). Fisher et al. (1985a) modelled the hydrodynamic and radiative response of the atmosphere to short impulsive injections of non-thermal electron beams. They showed that a high-energy flux of non-thermal electrons drives explosive evaporation accompanied by the formation of cool chromospheric condensations in the flare chromosphere.

A different situation occurs when the flux associated with non-thermal electrons is very low. Then only a weak chromospheric evaporation takes place. This kind of evaporation is referred to as gentle evaporation (Antiochos \& Sturrock 1978; 
Schmieder et al. 1987) and it can be observed in chromospheric spectral lines like $\mathrm{H} \alpha$ or in Ca II (8542 $\AA$ ). Antiochos $\&$ Sturrock suggested that the gentle chromospheric evaporation may occur after the primary energy release when the non-thermal electron flux is stopped. This evaporation could be driven by the large conductive heat flux from a hightemperature flare plasma contained in magnetic tubes above the chromosphere. Such physical conditions may appear during the gradual phase of solar flares, when there is no significant flux of non-thermal electrons. In the Forbes et al. (1989) model for flare-loop formation by magnetic reconnection the conduction of the thermal energy generated at the slow-mode shocks drives a gentle evaporative upflow from the ribbons. Schmieder et al. (1987) observed small but long-lasting blue shifts in flare ribbons in the $\mathrm{H} \alpha$ line during the gradual phase of three solar flares and interpreted them as due to upflows with velocities $<10 \mathrm{~km} \mathrm{~s}^{-1}$. These upflows were believed to be caused by gentle chromospheric evaporation driven by the heat conduction along the field lines connecting the chromosphere with a reconnection site in the corona. Extreme-ultraviolet (EUV) lines are also good indicators because the velocity can be derived more simply without solving the radiative transfer problem. Schmieder et al. (1990) were the first to detect an upflow in the EUV ribbons using data from the ultraviolet spectrometer and polarimeter (UVSP) on board the Solar Maximum Mission (SMM). These EUV upflows were confirmed by Czaykowska et al. (1999) observing flares during the gradual phase with the Coronal Diagnostic Spectrometer (CDS) on board SOHO.

There is wide agreement that the explosive evaporation occurs during the impulsive (flash) phase of the flares (e.g. Antonucci et al. 1984) and there are many examples derived from the X-ray line of Ca XIX. On the other hand, in the case of a gentle chromospheric evaporation observed during the gradual phase of solar flares there are still uncertainties concerning the spatial distribution of evaporating regions within the whole flare ribbons as well as the location of these regions with respect to X-ray loop footpoints where the heating can occur. Also the velocities of the evaporative plasma are not yet well determined. In particular, there is no information about the vertical structure of the velocity field in evaporative areas. Only Brosius (2003) gave some information about the Doppler velocities in several lines formed at different temperatures between $4.9<\log T<6.9$, as a function of time. From these data we can roughly deduce vertical dependence of the plasma velocity.

Schmieder et al. (1987) gave a qualitative interpretation of the $\mathrm{H} \alpha$ line shapes but a quantitative analysis based on radiative-transfer modelling is still missing. The only example of such a non-LTE modelling approach is given in Kašparova et al. (1998). The main difficulty in the analysis of such effects is the proper interpretation of the shape of the chromospheric line profiles. The evaporation observed during the impulsive phase of solar flares is manifested in the shape of X-ray spectral lines which are always in emission. The velocities of upflows are high and produce more asymmetric and shifted line profiles and thus it is easier to deduce the line-of-sight (LOS) velocity of the plasma. On the contrary, the gentle evaporation during the gradual phase is much slower and the $\mathrm{H} \alpha$ line profiles can be in absorption or enhanced emission. Very often we also observe self-reversed profiles. Deducing the velocity from such profiles is rather difficult and cannot be done only by searching for Doppler shifts with the bisector method (Heinzel et al. 1994). Chomospheric line profiles suggest that the flare atmosphere is highly dynamic and stratified with rather complicated plasma motion. The only reliable way to analyse the velocity field is to use the non-LTE transfer codes, which enable us to compute the chromospheric models with velocity fields. Resulting synthetic line profiles can then be compared with the observed ones.

In our present paper we construct a grid of flare models starting from the weak-flare model F1 of Machado et al. (1980) and using the non-LTE radiative transfer approach. Analysis of the $\mathrm{H} \alpha$ line profiles emitted by the flare is done by comparison with the synthetic line profiles calculated for a given velocity field. For the analysis we used the $\mathrm{H} \alpha$ line profiles of one solar flare obtained with the MSDP spectrograph mounted on the VTT telescope.

The paper is organised as follows. In Sect. 2 we present the observations of the analysed flare obtained on October 22, 2002. We also provide technical details of the line-profile reconstruction process. Section 3 presents the $\mathrm{H} \alpha$ line profiles obtained in the analysed areas of the flare. In Sect. 4 we describe the non-LTE radiative transfer codes used in our analysis and show how the codes are used to construct static and dynamic flare models. We also present some examples of synthetic $\mathrm{H} \alpha$ line profiles obtained from the models with a given velocity field. In Sect. 5 we describe the grids of models constructed to fit the observed profiles and we show the results of such fitting. Section 6 deals with the description and analysis of the velocity fields deduced from the line fitting procedure. In Sect. 7 we discuss our results, present our conclusions concerning the validity of our fitting procedure and interpret the results in the framework of the evaporative model of flares.

\section{Observations of the flare}

An M1.0 flare was observed in the active region NOAA 0162 on October 22, 2002, close to the solar disk centre (N26 E21, $\mu \simeq 0.8$ ). This active region was a target during a coordinated observational campaign between ground-based instruments (THEMIS and VTT) and space observatories (SOHO, TRACE and RHESSI). During its passage across the solar disk (17-31 October, 2002) the active region consisted of a large leading spot located in a positive magnetic field polarity region and a cluster of following spots of negative magnetic field polarity (Fig. 1). According to the GOES 9 flux curve, the flare onset was at 15:29 UT and the 1-8 $\AA$ X-ray flux reached its maximum at 15:36 UT (Fig. 2). During a long-duration decay phase, several local increases of the X-ray flux were detected, the most notable one with a peak at 16:28 UT.

$\mathrm{H} \alpha$ observations were performed using the MSDP (Multichannel Subtractive Double Pass) spectrograph (Mein 1991; Rompolt et al. 1993) coupled to the VTT telescope working at the Teide Observatory (Tenerife, Canary Islands). During the flare between 15:30 and 16:17 UT we completed 9 scans of the MSDP entrance window covering the whole active region 

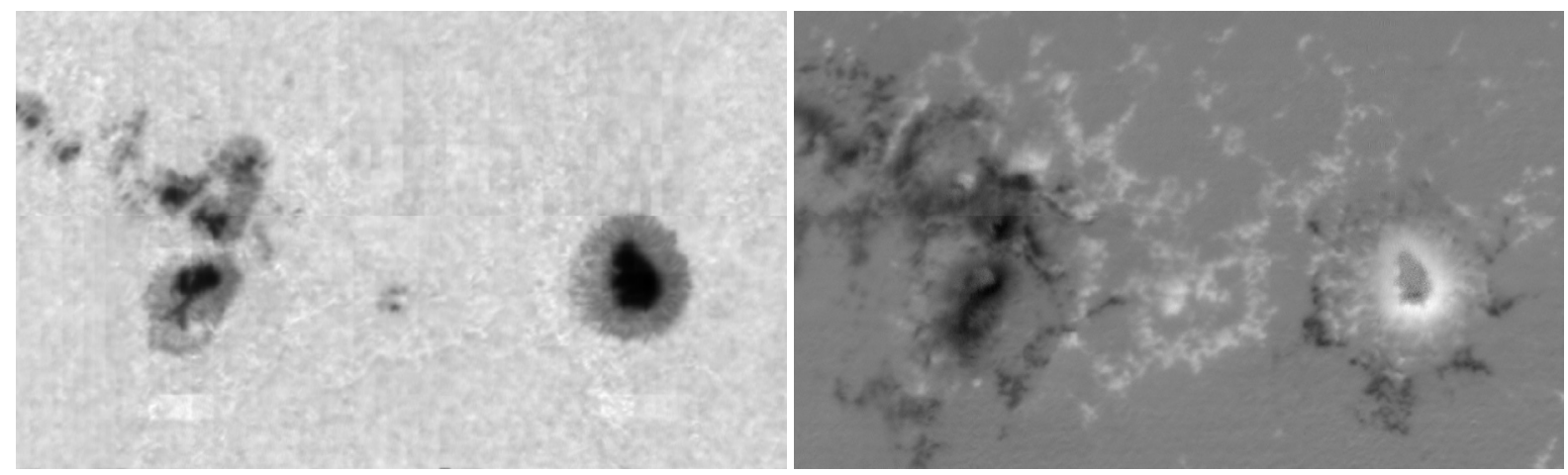

Fig. 1. Images of AR 0162 obtained simultaneously with the THEMIS/MSDP spectrograph between 15:41 and 16:29 UT by scanning. Left panel: $\mathrm{Na} \mathrm{I}_{1}$ line wing at $200 \mathrm{~m} \AA$ from the line centre. Right panel: line-of-sight (LOS) magnetic field deduced from $I$ and $V$ Stokes profiles at $100 \mathrm{~m} \AA$ from the $\mathrm{Na} \mathrm{I}_{1}$ line centre.

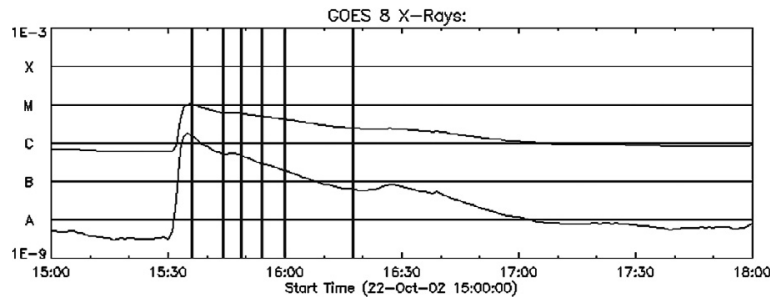

Fig. 2. Time evolution of GOES X-ray flux observed during the flare of October 22, 2002. Black vertical lines denote the approximate times of the VTT/MSDP images used in our analysis.

with a field-of-view (FOV) of about $380^{\prime \prime} \times 170^{\prime \prime}$. After processing the MSDP data we obtained monochromatic images which allowed us to reconstruct the $\mathrm{H} \alpha$ line profiles in all pixels of the image. The times of the MSDP observations (images) cited below correspond to the beginning of the appropriate scan, each scan lasting about $7 \mathrm{~min}$. The spatial resolution of the obtained images was limited by the seeing, on average it was about $1^{\prime \prime}$, but occasionally it was worse. Unfortunately, passing clouds caused changes of light intensity and for some scans we could not reconstruct images with the required quality. Therefore, we obtained only 7 full images at 15:30, 15:37, 15:44, 15:49, 15:54, 16:00 and 16:18 UT. The first image was taken during the impulsive phase thus we did not use it for the line-profile analysis. On the plot of the X-ray flux we marked with vertical lines the approximate times of the VTT/MSDP images used in our analysis (Fig. 2). All the analysed images were taken during the decay phase of the flare.

On the H $\alpha$ VTT/MSDP images, well developed flare ribbons were observed from 15:30 UT. The orientation of the two main ribbons $\mathrm{R} 1$ and $\mathrm{R} 2$ suggests that the AR is sheared (Fig. 3 - upper panel). The $\mathrm{H} \alpha$ ribbons seem to be shifted along the neutral line and they are not located opposite each other (Berlicki et al. 2004). The brightest parts of the $\mathrm{H} \alpha$ flare ribbons, are clearly visible at 15:30 UT, close to the black cross, but became much weaker after 7 min and almost disappeared at about 16:00 UT but other parts of the flare ribbons were still clearly visible (Fig. 3 - middle panel). At 15:30 UT the two brightest parts of the $\mathrm{H} \alpha$ ribbons were located in the following spots group, each of them in an area of magnetic field of opposite polarity (Fig. 3 - lower panel).
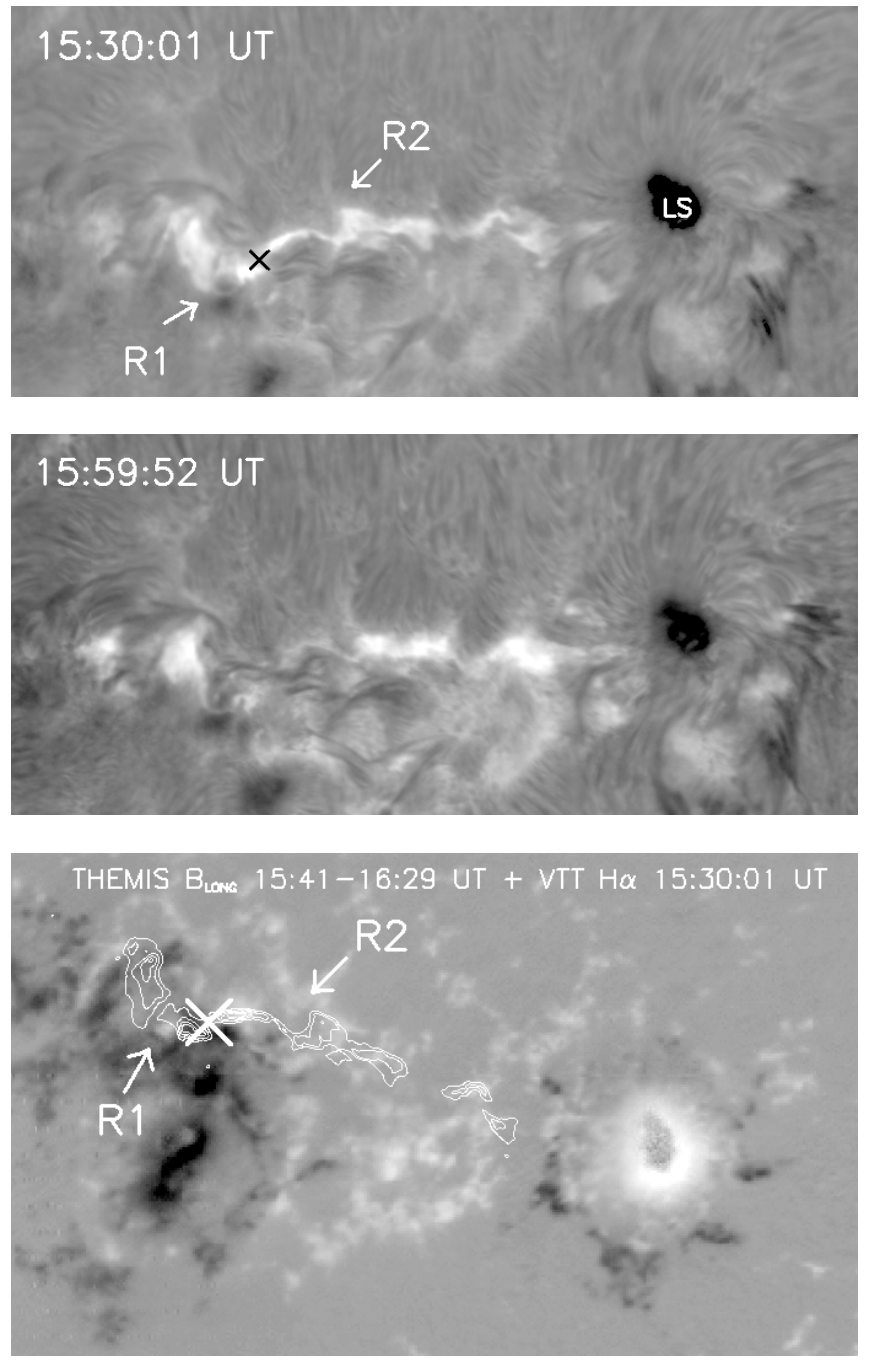

Fig. 3. The flare of October 22, 2002 observed in $\mathrm{H} \alpha$ line by MSDP/VTT spectrograph (upper and middle panel). The FOV is $440^{\prime \prime} \times 200^{\prime \prime}$. R1 and R2 denote two main ribbons of the flare and LS marks the leading spot of the AR 0162. In the lower panel we present line-of-sight (LOS) magnetic field deduced from $I$ and $V$ Stokes profiles overlaid with the contours of the $\mathrm{H} \alpha$ flare observed by VTT/MSDP at 15:30 UT. The field-of-view (FOV) is $400^{\prime \prime} \times 250^{\prime \prime}$. The crosses in the both images denote the place separating the two main ribbons $\mathrm{R} 1$ and $\mathrm{R} 2$. 


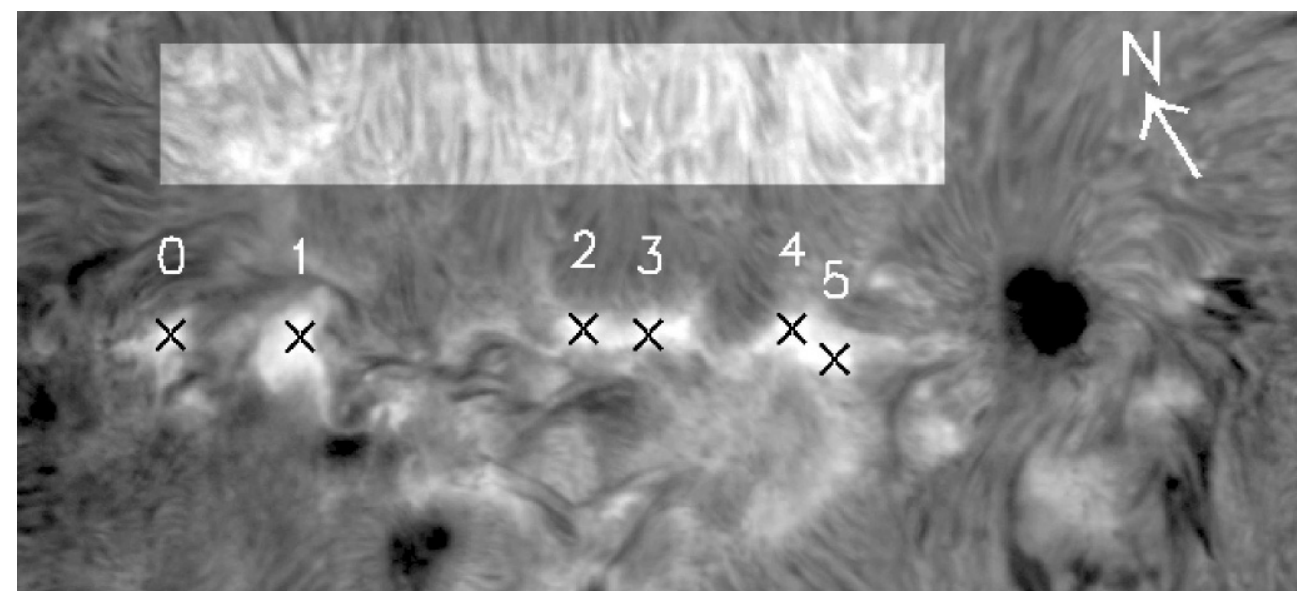

Fig. 4. VTT/MSDP image of AR 0162 made at 16:00 UT with crosses which mark the areas where the H $\alpha$ line profiles of the flare were obtained. The lighter rectangular area represents the region from which the mean $\mathrm{H} \alpha$ line profile of the quiet Sun was taken.

\section{Observed $\mathrm{H} \alpha$ line profiles}

For our analysis of the VTT/MSDP images we chose 6 areas where the observed $\mathrm{H} \alpha$ profiles were obtained. The black crosses marked on the MSDP image in Fig. 4 are the centres of the corresponding areas. In these areas the mean $\mathrm{H} \alpha$ line profiles were obtained from the MSDP spectral images.

For each pixel of the spectral images, the $\mathrm{H} \alpha$ line profiles are extracted automatically during the processing of MSDP data. In our analysis we obtained the mean profile for each analysed area by averaging the profiles coming from nine pixels contained inside a square box $0.75 \times 0.75^{\prime \prime}$, the distance between two neighbouring pixels being equal to $0.25^{\prime \prime}$. We chose six areas for the analysis in the VTT/MSDP image taken at 15:37 UT. The same areas were followed also in the next images taken at 15:44, 15:49, 15:54, 16:00 and 16:18 UT. Altogether, we used $36 \mathrm{H} \alpha$ line profiles (six areas at six different times). As an example we present in Fig. 5 the profiles observed in all analysed parts of the flare at 16:00 UT. The obtained profiles are typical for the weak-flare emission often observed during the late phases of solar flares. A rough inspection of the observed line profiles allows us to conclude that the structure of the chromosphere within flare ribbons changes during the considered range of time. In most cases we noticed a slow decrease of the $\mathrm{H} \alpha$ emission which indicates that the flaring chromosphere gradually approached that of the quiet Sun (Fig. 6). The $\mathrm{H} \alpha$ emission of the areas Nos. 4 and 5 slightly increases during the considered range of time because these parts of the flare became brighter later on. This is due to the propagation of the emission along the ribbon R2 towards the leading sunspot LS (Berlicki et al. 2004). All observed profiles are asymmetrical which indicates the presence of vertical motion in the chromosphere. We used the obtained profiles to derive the temperature and velocity structure of the flare in the six respective bright kernels.

\section{Non-LTE flare models}

We used the $\mathrm{H} \alpha$ line profiles described in the previous section to construct models of the solar chromosphere corresponding to the analysed areas of the flare. These observed line
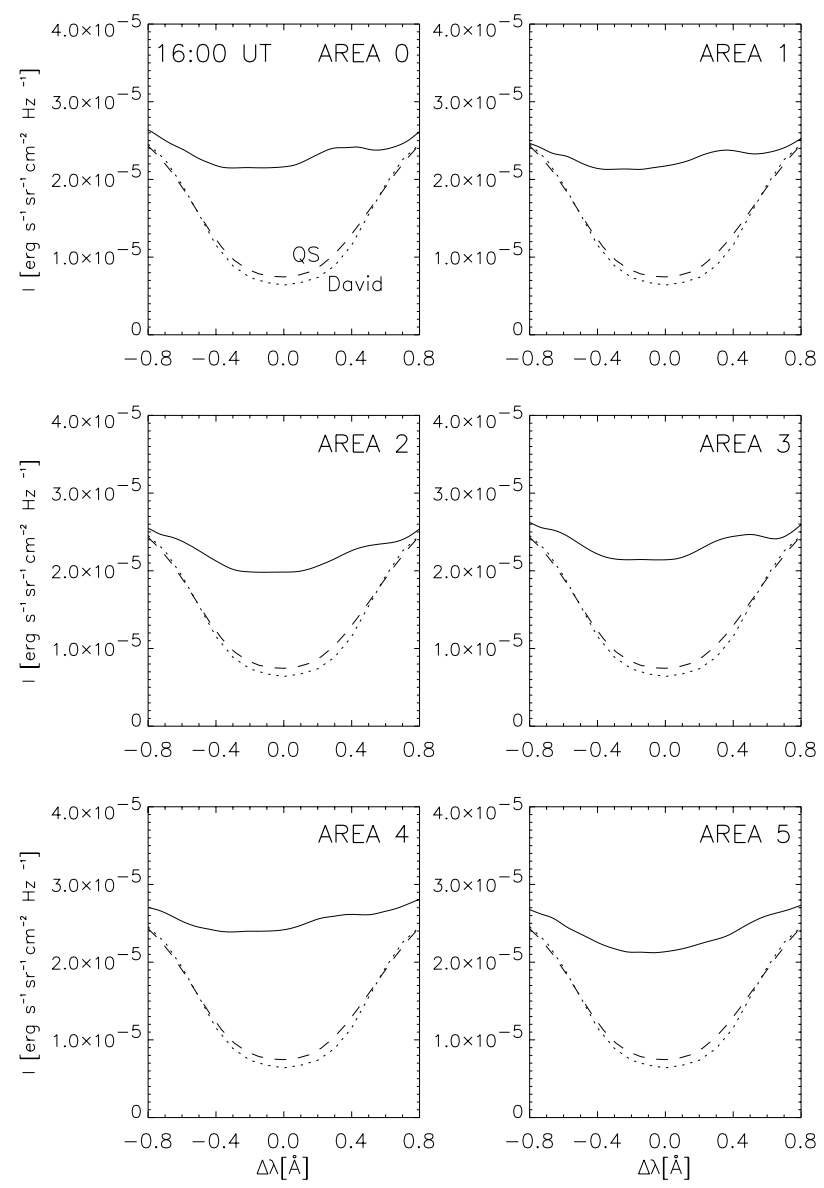

Fig. 5. H $\alpha$ line profiles observed by the MSDP/VTT spectrograph in analysed areas $0-5$ of the flare at 16:00 UT (continuous lines). Dotted lines denote the profile of the quiet-Sun chromosphere for $\mu=0.8$ (David 1961) and dashed lines represent the mean $\mathrm{H} \alpha$ line profile emitted by quiet-Sun region in the vicinity of the flare (see also Fig. 4).

profiles were compared with a grid of synthetic $\mathrm{H} \alpha$ line profiles calculated by the non-LTE code developed by Heinzel (1995) and modified for flare conditions. The code uses a 1D plane-parallel geometry and the atmosphere is in hydrostatic equilibrium. Hydrogen excitation and ionisation 


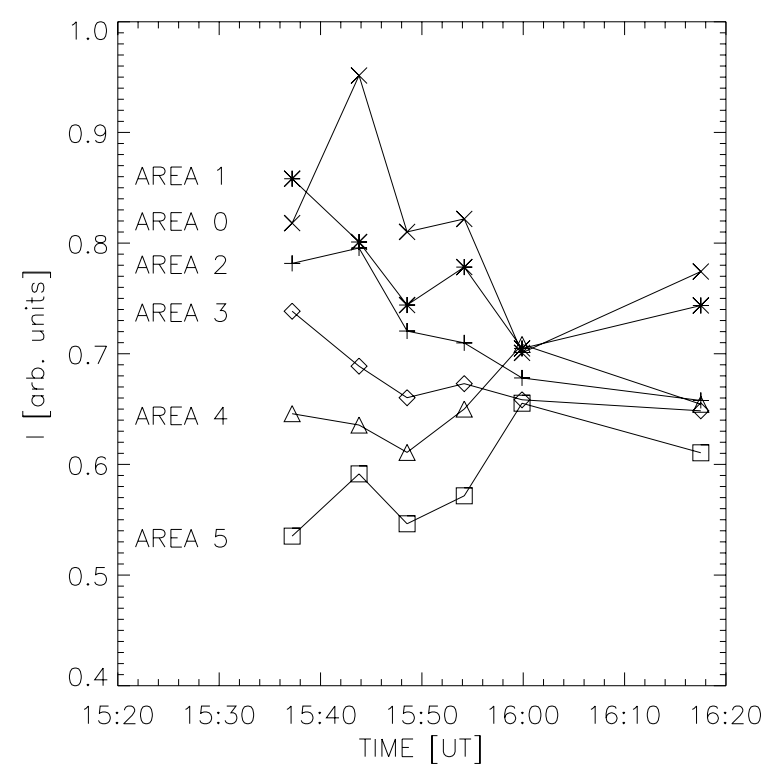

Fig. 6. Time evolution of the integral intensity of the $\mathrm{H} \alpha$ line profile contained within $\pm 0.7 \AA$ from the line centre plotted for all analysed areas.

equilibrium have been computed by solving simultaneously the radiative transfer equations, the equations of statistical equilibrium for a five-level plus continuum atomic model of hydrogen and the equations of particle and charge conservation. The equations of statistical equilibrium have been preconditioned according to Rybicki \& Hummer (1991). The preconditioning is based on the lambda-operator splitting technique, where the exact lambda operator is expressed as an approximate operator plus the correction. Then the correction is iteratively applied to a lagged source function by using the socalled Accelerated Lambda Iterations (ALI) method. For multilevel atoms this method is referred to as MALI - Multilevel Accelerated Lambda Iterations (Rybicki \& Hummer 1991). The preconditioned equations are then linearized with respect to the atomic level populations and electron density and solved iteratively (Heinzel 1995).

The code allows us to find a model of the flare chromosphere by varying two input parameters and using the semiempirical model F1 of a weak-flare (Machado et al. 1980) as an initial model (Berlicki \& Heinzel 2004). The input parameters used for modification of the reference atmospheric model F1 are $m_{0}$ (modification of the column mass scale of the reference atmosphere):

$m(\mathrm{ID})=m_{\mathrm{rm}}(\mathrm{ID})+m_{0}$,

and $\Delta T$ which modifies the temperature distribution of the reference atmosphere so that a new temperature is:

$T(\mathrm{ID})=T_{\mathrm{rm}}(\mathrm{ID})+\frac{\left[m_{1}-m(\mathrm{ID})\right] \Delta T}{m_{1}}$.

Subscript "rm" means the reference model (F1). The column mass $m_{1}$ determines the depth above which the temperature is modified (we took empirically $m_{1}=0.3$ ). ID is the depthpoint number in semiempirical models. Note that $m_{0}$ is related to variations of the coronal pressure at the top of our flare atmosphere.

To investigate the influence of the macroscopic velocity field on emergent $\mathrm{H} \alpha$ line profiles we adopted the formula used by Mihalas (1978) to describe an expanding atmosphere. The vertical velocity as a function of the $\mathrm{H} \alpha$ line-centre optical depth is defined as:

$V(\tau)=\frac{2 V_{0}}{1+\tau / \tau_{\mathrm{m}}}$

$\tau_{\mathrm{m}}$ corresponds to the height where the velocity is equal to $V_{0}$. Note that for $\tau \ll \tau_{\mathrm{m}}$, which corresponds to upper layers of the chromosphere, the velocity reaches $2 V_{0}$, and for $\tau \gg \tau_{\mathrm{m}}$ (close to the photosphere) the veloctiy goes to zero. Such a vertical structure of the velocity field seems to be a reasonable representation of an evaporating atmosphere. It was previously used in flaring atmosphere modelling (Nejezchleba 1998). In our paper we use the negative sign of the velocity for an upward motion. To simulate the effect of this velocity field on the emergent $\mathrm{H} \alpha$ line profiles, we performed some test calculations. In Fig. 7 we show profiles calculated for the F1 model with the velocity field described by $V_{0}=+8$ and $-8 \mathrm{~km} \mathrm{~s}^{-1}$ and $\log \tau_{\mathrm{m}}=0.1,1.0$ and 10.0 (see also Fig. 8).

It is noteworthy that for upflows the synthetic profiles show a red asymmetry while the line core is blue-shifted. This asymmetry depends on the velocities at corresponding lineformation depths.

Our procedure of fitting the $\mathrm{H} \alpha$ line profiles is the following. First, we constructed a grid of static models by varying two parameters $m_{0}$ and $\Delta T$. Each observed profile was fitted by the least-square technique to a closest synthetic profile from the static grid. Second, using the selected static model, we introduced the velocity field described by $V_{0}$ and $\tau_{\mathrm{m}}$ and performed a formal solution of the transfer equation to obtain an asymmetrical $\mathrm{H} \alpha$ profile. This was done for a range of values of $V_{0}$ and $\tau_{\mathrm{m}}$ so that for each selected static model we constructed another grid of synthetic profiles depending on velocities. Then the least-square method was used again to fit an observed asymmetrical profile. This two-step procedure saved us a significant amount of computing time because the full nonLTE solution was done only for the static models. Formal solutions for a grid of velocity fields are then very fast. However, this approach is justified only for relatively small velocities $\left(V \leq 10 \mathrm{~km} \mathrm{~s}^{-1}\right)$ which do not significantly affect the level populations (see Nejezchleba 1998). It cannot be used for impulsive phases when the chromospheric condensations move quite fast.

\section{Model analysis of the flaring chromosphere}

To fit all the observed line profiles with the synthetic ones, we computed a grid of $23 \times 26=598$ semiempirical static models by modifying the reference model F1 by changing the $m_{0}$ and $\Delta T$ parameters. We used 23 different values of $m_{0}$ (from $-10^{-4}$ to $+10^{-3}$, with step $5 \times 10^{-5} \mathrm{~g} \mathrm{~cm}^{-2}$ ) and 26 values of $\Delta T$ (from -700 to +550 with step $50 \mathrm{~K}$ ). For each model we obtained the synthetic profile of the $\mathrm{H} \alpha$ line (for $\mu=0.8$ ) taking into account the microturbulent and Stark broadening effects. 

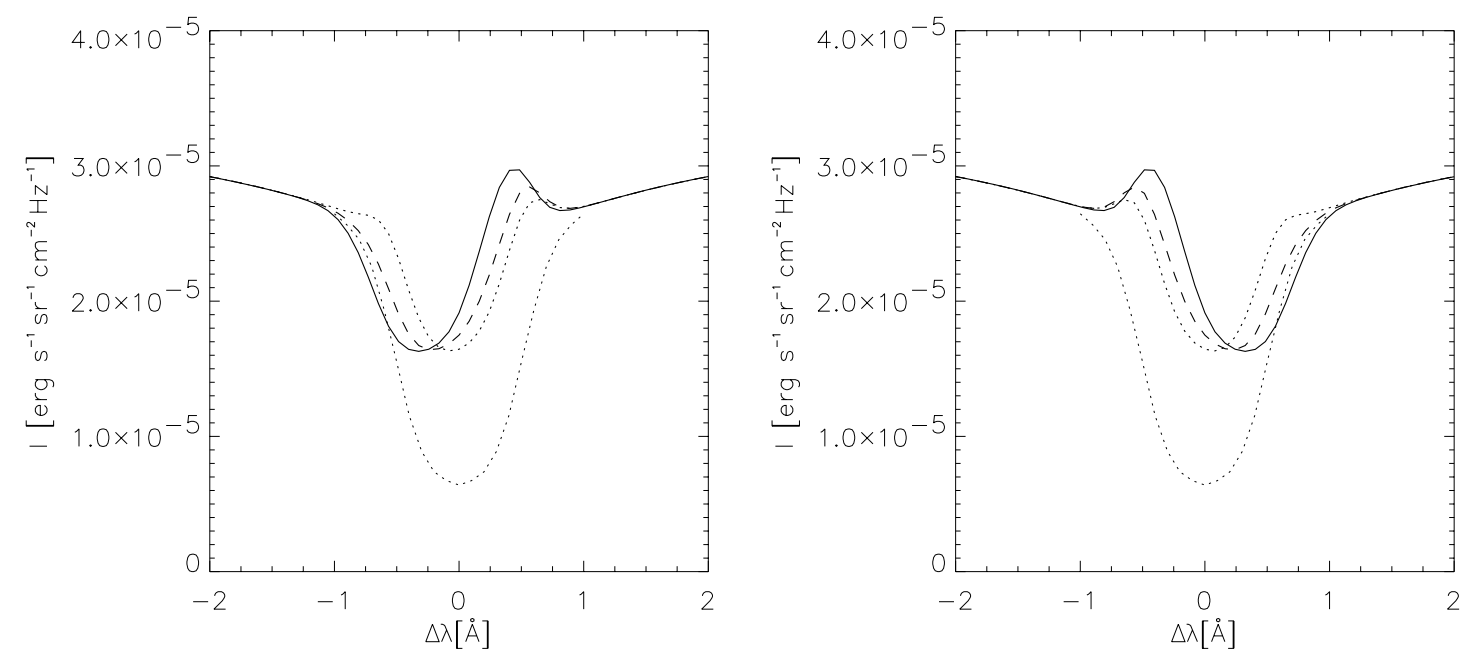

Fig. 7. Theoretical $\mathrm{H} \alpha$ line profiles calculated for an upflow defined by $V_{0}=-8$ (left panel) and downflow $V_{0}=+8 \mathrm{~km} \mathrm{~s}{ }^{-1}$ (right panel) for three values of $\log \tau_{\mathrm{m}}=0.1,1.0$ and 10.0 (dotted, dashed and continuous lines, respectively). The calculations were performed for the weakflare model atmosphere F1. For comparison, we plotted the H $\alpha$ line profile (David 1961) of the quiet-Sun chromosphere (lower dotted line).
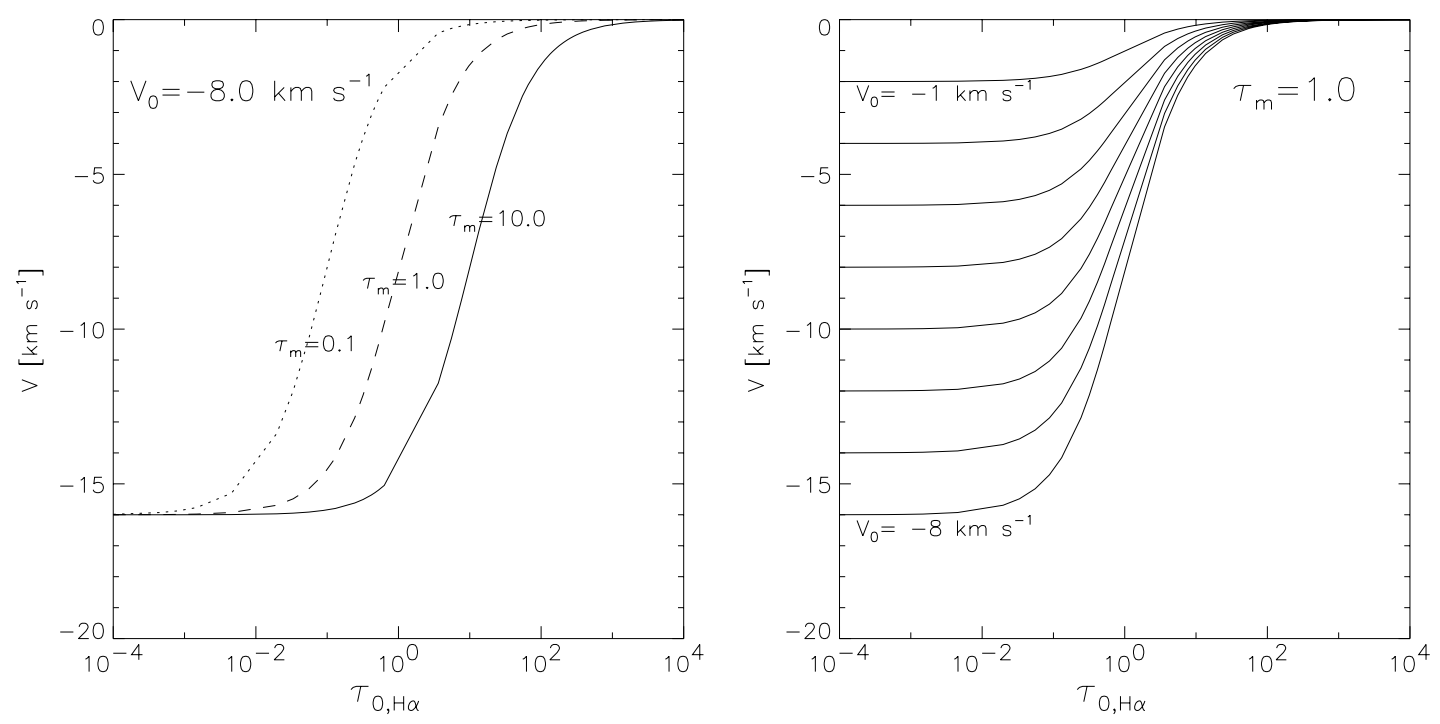

Fig. 8. The $\mathrm{H} \alpha$ line-centre optical depth distribution of the velocity in a flare atmosphere for different values of $\tau_{\mathrm{m}}$ and for $V_{0}=-8 \mathrm{~km} \mathrm{~s}$ (left panel) and for different values of $V_{0}$ and for $\tau_{\mathrm{m}}=1.0$ (right panel). In construction of the grid of models with the velocity field $\tau_{\mathrm{m}}$ varies from 0.1 to 10.0 and $V_{0}$ from -8 to $+8 \mathrm{~km} \mathrm{~s}^{-1}$.

Next, we have convolved all these profiles with a Gaussian profile of half-width $0.20 \AA$ (equivalent to $7 \mathrm{~km} \mathrm{~s}^{-1}$ at the $\mathrm{H} \alpha$ line wavelength). This value was found empirically and the aim of this procedure was to simulate the macroturbulent broadening and the instrumental effects of the MSDP instrument.

For each $\mathrm{H} \alpha$ profile observed in the analysed areas of the flare, we found the best fit among the static synthetic profiles. In the fitting procedure for each observed profile we found the differences for a given observed profile $I^{\text {obs }}$ and all 598 synthetic profiles $I^{\text {synth }}$ in $120 \lambda_{i}$-points located between -0.6 and $+0.6 \AA$ from the $\mathrm{H} \alpha$ line centre. We defined this difference for the $n$-th synthetic profile as:

$\chi_{n}^{2}=\sum_{i=1}^{120}\left(I_{\lambda_{i}}^{\mathrm{obs}}-I_{n, \lambda_{i}}^{\mathrm{synth}}\right)^{2}$, for $n=1, \ldots, 598$
Then, among all values of $\chi_{n}^{2}$ the minimum was found. In this way, we determined the static model of the chromosphere within each analysed area at all times. The parameters of these models are given in Table 1.

In the next step we introduced the velocity field dependent on the $\mathrm{H} \alpha$ line-centre optical depth computed in the given static model. The atomic level populations and electron densities come from the previously computed static models, but the $\mathrm{H} \alpha$ line profile is Doppler-shifted due to flows. In this way, for each analysed area, we constructed another grid of $21 \times 33=693$ models with velocity fields. We used 21 different values of the parameters $\log \tau_{\mathrm{m}}$ (from -1.0 to +1.0 , step 0.1 ) and 33 values of $V_{0}$ (from -8.0 to +8.0 step $0.5 \mathrm{~km} \mathrm{~s}^{-1}$ ). These parameters are then used in Eq. (3) to define the vertical structure of the velocity field in the flaring chromosphere. Note that if we computed fully dynamic non-LTE models instead of static 
Table 1. The parameters $m_{0}$ and $\Delta T$ of the static models deduced from the fitting of synthetic $\mathrm{H} \alpha$ line profiles with observed ones.

\begin{tabular}{|c|c|c|c|}
\hline Area & Time[UT] & $m_{0}\left[\mathrm{~g} \mathrm{~cm}^{-2}\right]$ & $\Delta T[\mathrm{~K}]$ \\
\hline \multirow{6}{*}{0} & $15: 37$ & $1.0 \times 10^{-3}$ & -350 \\
\hline & $15: 44$ & $1.0 \times 10^{-3}$ & -300 \\
\hline & $15: 49$ & $8.5 \times 10^{-4}$ & -350 \\
\hline & $15: 54$ & $7.5 \times 10^{-4}$ & -400 \\
\hline & $16: 00$ & $5.5 \times 10^{-4}$ & -500 \\
\hline & $16: 18$ & $3.0 \times 10^{-4}$ & -650 \\
\hline \multirow{6}{*}{1} & $15: 37$ & $1.0 \times 10^{-3}$ & -100 \\
\hline & $15: 44$ & $1.0 \times 10^{-3}$ & -400 \\
\hline & $15: 49$ & $8.0 \times 10^{-4}$ & -300 \\
\hline & $15: 54$ & $5.5 \times 10^{-4}$ & -400 \\
\hline & $16: 00$ & $6.0 \times 10^{-4}$ & -550 \\
\hline & $16: 18$ & $4.0 \times 10^{-4}$ & -550 \\
\hline \multirow{6}{*}{2} & $15: 37$ & $9.5 \times 10^{-4}$ & -350 \\
\hline & $15: 44$ & $7.0 \times 10^{-4}$ & -350 \\
\hline & $15: 49$ & $6.5 \times 10^{-4}$ & -350 \\
\hline & $15: 54$ & $4.5 \times 10^{-4}$ & -350 \\
\hline & $16: 00$ & $3.5 \times 10^{-4}$ & -400 \\
\hline & $16: 18$ & $2.5 \times 10^{-4}$ & -550 \\
\hline \multirow{6}{*}{3} & $15: 37$ & $1.0 \times 10^{-3}$ & -350 \\
\hline & $15: 44$ & $1.0 \times 10^{-3}$ & -450 \\
\hline & $15: 49$ & $6.5 \times 10^{-4}$ & -400 \\
\hline & $15: 54$ & $4.5 \times 10^{-4}$ & -350 \\
\hline & $16: 00$ & $4.5 \times 10^{-4}$ & -400 \\
\hline & 16:18 & $3.0 \times 10^{-4}$ & -500 \\
\hline \multirow{6}{*}{4} & $15: 37$ & $7.0 \times 10^{-4}$ & -450 \\
\hline & $15: 44$ & $7.0 \times 10^{-4}$ & -450 \\
\hline & $15: 49$ & $5.5 \times 10^{-4}$ & -400 \\
\hline & $15: 54$ & $5.0 \times 10^{-4}$ & -400 \\
\hline & $16: 00$ & $7.0 \times 10^{-4}$ & -450 \\
\hline & $16: 18$ & $5.0 \times 10^{-4}$ & -450 \\
\hline \multirow{6}{*}{5} & $15: 37$ & $7.5 \times 10^{-4}$ & -300 \\
\hline & $15: 44$ & $7.0 \times 10^{-4}$ & -350 \\
\hline & $15: 49$ & $4.5 \times 10^{-4}$ & -400 \\
\hline & $15: 54$ & $4.5 \times 10^{-4}$ & -400 \\
\hline & $16: 00$ & $4.0 \times 10^{-4}$ & -350 \\
\hline & 16:18 & $3.5 \times 10^{-4}$ & -400 \\
\hline
\end{tabular}

ones, we should construct a grid of $598 \times 693=414414$ models (few months of CPU time on a fast computer).

To illustrate the nature of the velocity field as defined in our models we present in Fig. 8 the depth distribution of the velocity in a flare atmosphere for different values of $\tau_{\mathrm{m}}$ and $V_{0}$. From the left panel of Fig. 8 it follows that for a given $V_{0}$ parameter the velocity at the depth where the centre of the $\mathrm{H} \alpha$ line is formed $\left(\tau_{0, \mathrm{H} \alpha} \approx 1\right)$ increases with increasing $\tau_{\mathrm{m}}$. Therefore, the profiles obtained for models with large values of $\tau_{\mathrm{m}}$ should be more asymmetric.

For all of the models with the velocity field, we obtained synthetic profiles of the $\mathrm{H} \alpha$ line and we repeated the convolution with a Gaussian function of the same half-width as previously. Finally, for each $\mathrm{H} \alpha$ profile observed in the analysed areas of the flare, we found the best fit among these synthetic
Table 2. Optical depth $\log \tau_{\mathrm{m}}$ and the vertical velocity parameter $V_{0}$ of the models obtained from the fitting procedure.

\begin{tabular}{|c|c|c|c|}
\hline Area & Time[UT] & $\log \tau_{\mathrm{m}}$ & $V_{0}\left[\mathrm{~km} \mathrm{~s}^{-1}\right]$ \\
\hline \multirow{6}{*}{0} & $15: 37$ & - & 0.0 \\
\hline & $15: 44$ & -0.2 & -10.0 \\
\hline & $15: 49$ & - & 0.0 \\
\hline & $15: 54$ & +1.0 & -1.2 \\
\hline & $16: 00$ & +0.7 & -6.9 \\
\hline & $16: 18$ & +0.6 & -1.9 \\
\hline \multirow{6}{*}{1} & $15: 37$ & -0.2 & -10.0 \\
\hline & $15: 44$ & -1.0 & +0.6 \\
\hline & $15: 49$ & -0.7 & -5.6 \\
\hline & $15: 54$ & -0.8 & -5.0 \\
\hline & $16: 00$ & +0.5 & -8.7 \\
\hline & $16: 18$ & +0.1 & +1.9 \\
\hline \multirow{6}{*}{2} & $15: 37$ & -0.4 & -3.7 \\
\hline & $15: 44$ & -0.3 & -4.4 \\
\hline & $15: 49$ & -0.9 & +0.6 \\
\hline & $15: 54$ & +0.5 & -1.9 \\
\hline & $16: 00$ & +1.0 & -1.9 \\
\hline & $16: 18$ & -0.1 & -1.2 \\
\hline \multirow{6}{*}{3} & $15: 37$ & -0.3 & -10.0 \\
\hline & $15: 44$ & -1.0 & +5.6 \\
\hline & $15: 49$ & +1.0 & -5.0 \\
\hline & $15: 54$ & -0.2 & -1.9 \\
\hline & $16: 00$ & +1.0 & -3.7 \\
\hline & $16: 18$ & -0.8 & -0.6 \\
\hline \multirow{6}{*}{4} & $15: 37$ & +0.4 & -4.4 \\
\hline & $15: 44$ & +0.4 & -1.2 \\
\hline & $15: 49$ & -1.0 & -7.5 \\
\hline & $15: 54$ & 0.0 & +0.6 \\
\hline & $16: 00$ & +0.3 & -10.0 \\
\hline & $16: 18$ & -0.5 & -10.0 \\
\hline \multirow{6}{*}{5} & $15: 37$ & +0.6 & -1.2 \\
\hline & $15: 44$ & +0.1 & -1.9 \\
\hline & $15: 49$ & -0.1 & -1.2 \\
\hline & $15: 54$ & +1.0 & -0.6 \\
\hline & $16: 00$ & -0.2 & -3.1 \\
\hline & $16: 18$ & -1.0 & +1.9 \\
\hline
\end{tabular}

profiles. The fitting procedure was similar to the one used for the static models, i.e. searching for the $\chi^{2}$-minimum in the whole grid of profiles. In this way we found the semiempirical models and the height distribution of the velocity within all analysed areas of the flare. The parameters describing the velocity field found in the analysed areas are given in Table 2 . We corrected the vertical velocity parameter $V_{0}$ with the value of the direction cosine $\mu=0.8$ and we assumed that the velocity vector is perpendicular to the solar surface. As an example in Fig. 9 we show the vertical structure of the velocity field for the area No. 5 as a function of the $\mathrm{H} \alpha$ line-centre optical depth $\tau_{0, \mathrm{H} \alpha}$. The scheme shows principally upflow during all the period of observations. Nevertheless, downflow is observed late in the gradual phase at 16:18 UT. 


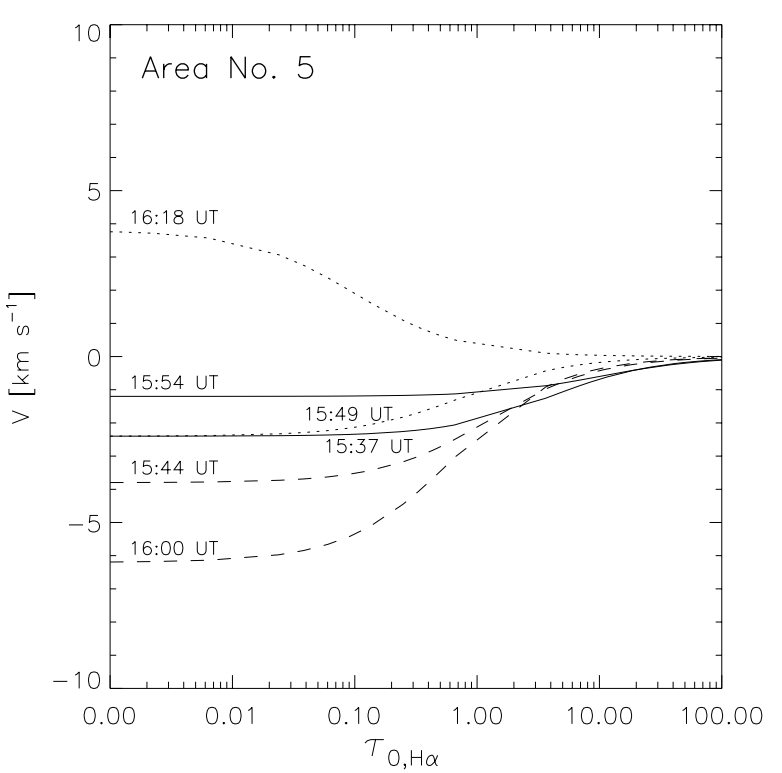

Fig. 9. Vertical velocity of the plasma as a function of the $\mathrm{H} \alpha$ linecentre optical depth $\tau_{0, \mathrm{H} \alpha}$ determined for the area No. 5 at six times. At all times except 16:18 UT we observe upflows.

\section{Characteristics of the velocity field}

In most of the analysed areas and at most of the times the velocites obtained from the modelling exhibit negative values which means upward flows. In Fig. 10 we present the time evolution of the $\mathrm{H} \alpha$ line profiles observed in areas 2 and 5 and the synthetic profiles obtained from the fitting procedure which gave us the velocity fields.

Most of the profiles obtained in the analysed areas of the flare ribbons exhibit a red asymmetry manifested by the increase of the intensity in the red wing around 0.4-0.5. . As we can see from the test calculations (Fig. 7), in the case of self-reversed profiles the red asymmetry means an upflow. From Table 2, among all 36 analysed line profiles, 2 of them indicated no velocity, in 6 of them we noticed downflows, and from 28 line profiles we found upward flows. The derived $V_{0}$ parameter is within the range -10.0 and $+5.6 \mathrm{~km} \mathrm{~s}^{-1}$. The parameter $\tau_{\mathrm{m}}$ covers the full range from 0.1 to 10.0 . The range of the temporal changes of $V_{0}$ is different in each analysed area (Fig. 11). In the areas Nos. 2 and 5 the velocities are contained in a rather narrow range, between -4.4 and $+0.6 \mathrm{~km} \mathrm{~s}^{-1}$ for area No. 2, and between -3.1 and $+1.9 \mathrm{~km} \mathrm{~s}^{-1}$ for area No. 5 (Fig. 11 - right panel). This suggests that the fitting of the observed profiles performed for these areas was of rather good quality. The parameter $V_{0}$ determined for areas Nos. 1 and 4 varies much more with time (between -10.0 and $+1.9 \mathrm{~km} \mathrm{~s}^{-1}$ for area No. 1, and between -10.0 and $+0.6 \mathrm{~km} \mathrm{~s}^{-1}$ for area No. 4) which may indicate a less precise fitting (Fig. 11 - right panel). From the tests performed for many profiles it follows that the quality of the fitting depends on the shape of the observed profile to be fitted. Some profiles are very flat which largely complicates their fitting.

In Fig. 12 (left panel) we present a cross-correlation between the accuracy of the fitting represented by the value of $\chi^{2}$ parameter and the value of $V_{0}$. Some of the values of $V_{0}$ obtained from the profiles correspond to high values of $\chi^{2}$. If we assume that the velocities associated with $\chi^{2} \leq$ $2-3 \times 10^{11}$ are obtained with reasonable accuracy then from Fig. 12 (left panel) we see evident predominance of upflows. High values of $\chi^{2}$ are associated with the profiles, the shape of which cannot be reliably reproduced by our codes. For example, the velocity found in area No. 0 at 15:44 UT is equal $-10.0 \mathrm{~km} \mathrm{~s}^{-1}$ but the accuracy of fitting is low $\left(\chi^{2}=7 \times 10^{11}\right)$. To see if there is some relationship between the value of the velocity in the chromosphere and the optical depth, we present in the right panel of Fig. 12 the relationship between the parameters $V_{0}$ and $\tau_{\mathrm{m}}$ obtained for all the analysed profiles. We do not see any significant correlation between these two parameters and all the points are distributed more or less uniformly within the whole range of $\tau_{\mathrm{m}}(0.1-10.0)$.

\section{Discussion and conclusions}

In this paper we studied the velocity field in the solar flaring chromosphere. For the first time the evaporative flows in the gradual phase are studied quantitatively by using a non-LTE radiative transfer code. To analyse the vertical motion of the plasma within the flare ribbons we used spectral observations from the VTT/MSDP of a solar flare on October 22, 2002. The flare occurred in AR NOAA 0162, which at this time was located relatively close to the solar disk centre, giving us the possibility of obtaining valuable $\mathrm{H} \alpha$ line profiles of the flare emission used in the modelling of the velocity field with nonLTE radiative transfer codes (Heinzel 1995). For this purpose the observed $\mathrm{H} \alpha$ line profiles obtained in chosen areas of the flare ribbons were extracted from MSDP images. These profiles were then used in the fitting procedure where we compared them with grids of synthetic profiles calculated by the non-LTE codes. We constructed two grids: static and with a prescribed velocity field. From the comparison of observed and synthetic profiles we found models of the solar chromosphere in different analysed parts of the flare ribbons with the velocity field as a function of the $\mathrm{H} \alpha$ optical depth.

In our analysis we chose six different areas analysed at six different times during the gradual phase of the flare. In most of the cases the vertical velocities obtained from the fitting procedure were negative which indicates upflows. These results are in agreement with previous qualitative works (Schmieder et al. 1987). The time dependence of the velocity does not show any significant changes. Only in area No. 3 the deduced velocity changes from $-10.0 \mathrm{~km} \mathrm{~s}^{-1}$ upflow to $+5.6 \mathrm{~km} \mathrm{~s}^{-1}$ downflow and again to $-5.0 \mathrm{~km} \mathrm{~s}^{-1}$ upflow but the velocity of $+5.6 \mathrm{~km} \mathrm{~s}^{-1}$ is obtained with a large value of $\chi^{2}$ (Fig. 12 - left panel) and it may be unrealistic. These upflows concern the plasma at chromospheric levels. The optical-depth dependence of the velocity assumed in our modelling is such that the velocity increases with the height above the photosphere. At the top of the chromosphere, close to the chromosphere-corona transition region (CCTR), the vertical velocities are maximal and reach the value $2 V_{0}$. Thus, we can expect that in the CCTR and low corona the plasma flows should also be observed, even with higher velocities. Such upflows were reported by Schmieder et al. (1990) 

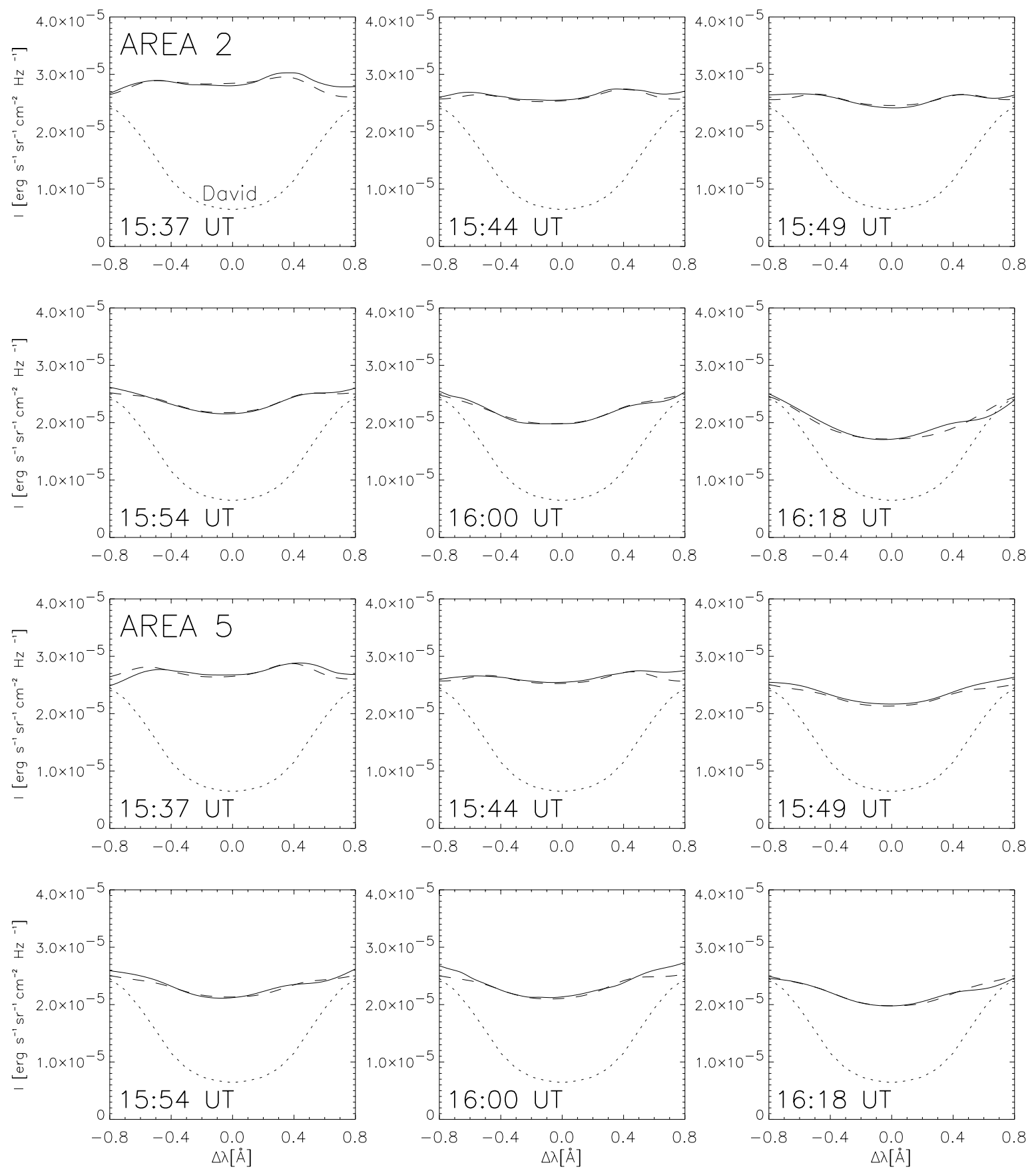

Fig. 10. The observed (continuous lines) and fitted (dashed lines) $\mathrm{H} \alpha$ line profiles in areas Nos. 2 and 5 of the flare. With dotted line we also plotted the mean profile of the quiet-Sun chromosphere taken from David (1961) for $\mu=0.8$.

and Czaykowska et al. (1999). In the first paper the authors report upflows in the range of $4-12 \mathrm{~km} \mathrm{~s}^{-1}$ deduced from EUV C IV $1548 \AA$ transition-region lines observed by the SMM/UVSP instrument. These velocities are consistent with the values found for the $\mathrm{H} \alpha$ line in our analysis. Czaykowska et al. (1999) used SOHO/CDS observations performed in a few EUV lines and found chromospheric upflows in the CCTR occurring above the $\mathrm{H} \alpha$ flare ribbons with velocities up to $100 \mathrm{~km} \mathrm{~s}^{-1}$ in Fe XVI and $20 \mathrm{~km} \mathrm{~s}^{-1}$ in $\mathrm{O} \mathrm{V}$ lines. Fe XVI line is formed at much higher temperatures and therefore higher in the corona. Thus, the larger velocities observed in this line are in agreement with our result if we assume the expanding character for the velocity structure in the flare atmosphere. It seems that from the observations of Czaykowska et al. (1999) the velocity of evaporating plasma increases with the temperature of the line formation.

We interpret the upflows found in the flare ribbons in terms of the Antiochos and Sturrock (1978) model for gentle evaporation. This process may occur during the gradual phase of solar flares and it can be driven by conductive heat flux from the high-temperature flare plasma contained in magnetic flux tubes above the photosphere. In the Forbes et al. (1989) model for flare-loop formation by magnetic reconnection the thermal energy is provided by slow-mode shocks produced by magnetic 

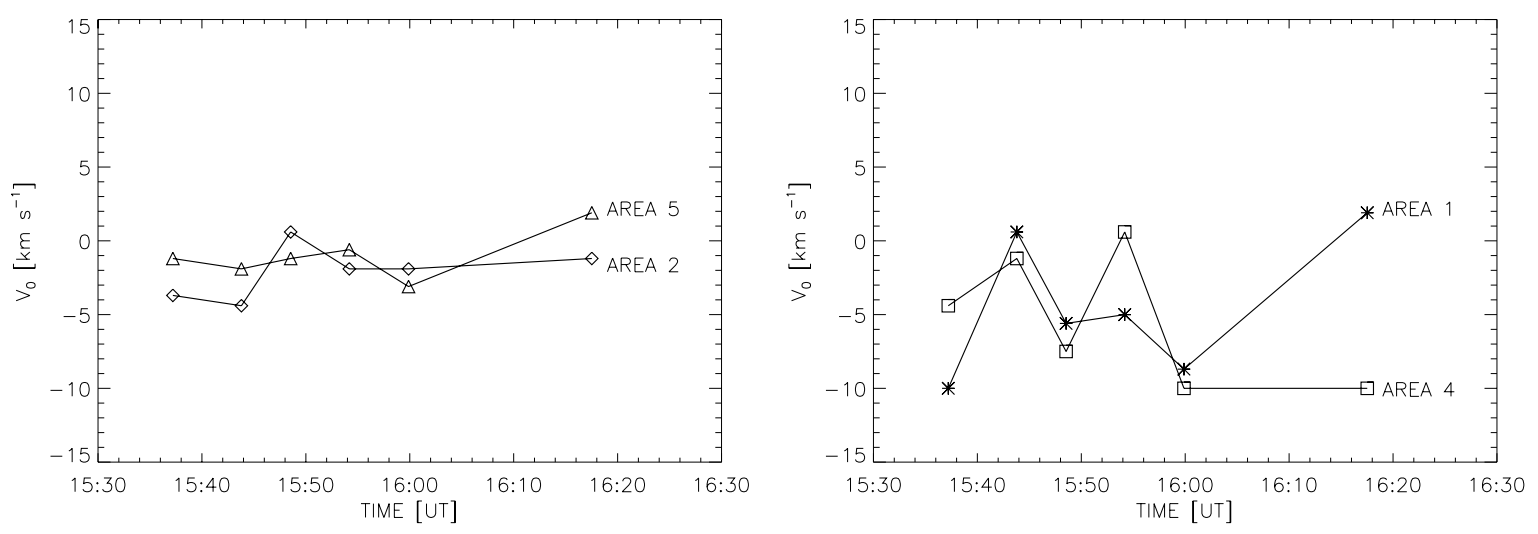

Fig. 11. Time dependence of the velocity $V_{0}$ determined from the fitting procedure in areas Nos. 2 and 5 (left panel) and in areas Nos. 1 and 4 (right panel).
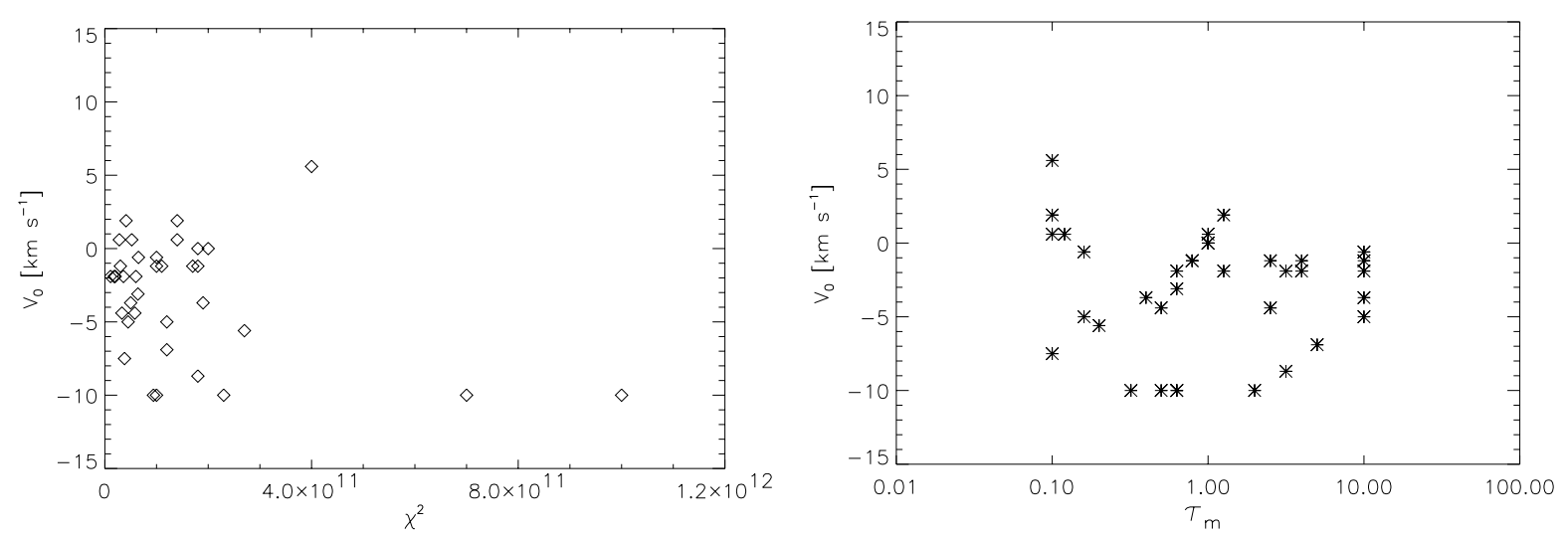

Fig. 12. Cross-correlation between the velocity parameter $V_{0}$ and the accuracy of the fitting described by $\chi^{2}$ (left panel) and the optical depth parameter $\tau_{\mathrm{m}}$ (right panel).

reconnection high in the corona. The conduction of the thermal energy generated at the slow shocks locations drives a gentle evaporative upflow from the ribbons whose widths are determined by the distance between the X-line fast-mode termination shock (Forbes et al. 1989). The predicted upflow velocity is less than $20 \mathrm{~km} \mathrm{~s}^{-1}$ in the CCTR region (Schmieder et al. 1990). Our results are consistent with this model. An interesting result comes from Table 1 . For all areas, the $m_{0}$ parameter is decreasing with time. This indicates that the evaporation gradually decreases with time, loading less mass into the coronal loops. In these areas the intensity of the $\mathrm{H} \alpha$ flare ribbons also decreases with time.

In this analysis, the main problem occurs in the fitting procedure. The flaring chromosphere is very dynamic and it is difficult to predict the velocity field at all heights. In our modelling we parameterise the velocity field only with two parameters $V_{0}$ and $\tau_{\mathrm{m}}$. In this way we assume a given function describing the vertical velocity, where the velocity is maximal at the top of the chromosphere and goes to zero at photospheric levels. This approach seems to be reasonable from a dynamical point of view. The density of the plasma is much lower at the top of the chromosphere and from a stationary equation of continuity one gets larger velocities as compared to the base of the chromosphere where the densities are much higher. The formula (Eq. (3)) used to define the vertical structure of the velocity characterises the overall structure of the velocity field but it cannot predict any peculiarities in the velocity field. Therefore, the synthetic profiles calculated with this formula can depart from the observed ones and for some of them the fitting procedure gives less accurate results. Using a more complicated definition of the velocity field is also possible, but to calculate the grid of models (and resulting profiles) which covers a wide range of parameters would be much more demanding. Nevertheless, the most important result is that with our rather simple formula we could reproduce the profiles with the red asymmetry, similar to most of the observed ones. This then indicates the upflows.

Another reason for difficulties in the fitting procedure was the narrow wavelength range of the obtained MSDP $\mathrm{H} \alpha$ profiles. The MSDP-type spectrograph has an advantage that we can obtain the line profiles in each pixel of a large FOV. However, the spectral range of the profiles is limited by instrumental considerations. In our observations we were only able to reconstruct the profiles within $\pm 0.9 \AA$. Therefore, we could not use the line wings to perform better calibrations and fitting of the whole profiles.

Taking into account all these remarks, in the future it would be interesting to use more spatial points at more times and to use the spectra obtained within a wider range of wavelengths. Other distributions of the velocity field in the chromosphere should also be tested. In addition, to perform non-LTE modelling of the flare structure it would be useful to have other 
spectral lines formed at different levels of the chromosphere like hydrogen $\mathrm{H} \beta, \mathrm{H} \gamma$, infrared calcium line (Ca II $8542 \AA$ ) etc.

Acknowledgements. This research was supported by the European Commission through the RTN programme ESMN (European Solar Magnetism Network, contract HPRN-CT-2002-00313). The authors are members of ESMN. This work was also partially supported by the grant A3003203 of the Grant Agency of the Academy of Sciences of the Czech Republic. P.H. acknowledges support of the Observatoire de Paris during his stay in Meudon. VTT/MSDP observations have been obtained in cooperation with J. Staiger. We also would like to thank the THEMIS team who operates the telescope at Tenerife. We thank the anonymous referee for comments which improved the paper.

\section{References}

Antiochos, S., \& Sturrock, P. 1978, ApJ, 220, 1137

Antonucci, E., Gabriel, A., \& Dennis, B. 1984, ApJ, 287, 917

Berlicki, A., Rudawy, P., Siarkowski, M., \& Jurecki, M. 2002, Adv. Space Res., 30, 605

Berlicki, A., \& Heinzel, P. 2004, A\&A, 420, 319

Berlicki, A., Schmieder, B., Vilmer, N., Aulanier, G., \& Del Zanna, G. 2004, A\&A, 423, 1119

Brosius, J. W. 2003, ApJ, 586, 1417

Czaykowska, A., De Pontieu, B., Alexander, D., \& Rank, G. 1999, ApJ, 521, 75

David, K. H. 1961, ZAp, 53, 37

Doschek, G. A., Mariska, J. T., Strong, K. T., et al. 1994, ApJ, 431, 888

Fisher, G., Canfield, R., \& McClymont, A. 1985a, ApJ, 289, 414
Fisher, G., Canfield, R., \& McClymont, A. 1985b, ApJ, 289, 434

Fisher, G. 1987, ApJ, 317, 502

Forbes, T. G., Malherbe, J. M., \& Priest, E. R. 1989, Sol. Phys., 120, 285

Heinzel, P., Karlický, M., Kotrč, P., \& Švestka, Z. 1994, Sol. Phys., 152,393

Heinzel, P. 1995, A\&A, 299, 563

Ichimoto, K., \& Kurokawa, H. 1984, Sol. Phys., 93, 105

Ji, H., Wang, H., Schmahl, E. J., Qiu, J., \& Zhang, Y. 2004, ApJ, 605, 938

Kašparova, J., Kotrc, P., Heinzel, P., Nikulin, I. F., \& Rudawy, P. 1998, IAU Coll., 167, ASP Conf. Ser., 1150

Kopp, R. A., \& Pneuman, G. W. 1976, Sol. Phys., 50, 85

Machado, M. E., Avrett, E. H., Vernazza, J. E., \& Noyes, R. W. 1980, ApJ, 242, 336

Mein, P. 1991, A\&A, 248, 669

Mihalas, D. 1978, Stellar Atmospheres, 2nd ed. (San Francisco: W. H. Freeman)

Nejezchleba, T. 1998, A\&AS, 127, 607

Rompolt, B., Mein, P., Mein, N., Rudawy, P., \& Berlicki, A. 1993, JOSO Annual Report 1993, 87

Rybicki, G. B., \& Hummer, D. 1991, A\&A, 245, 171

Schmieder, B., Forbes, T. G., Malherbe, J. M., \& Machado, M. E. 1987, ApJ, 317, 956

Schmieder, B., Malherbe, J. M., Simnett, G. M., Forbes, T. G., \& Tandberg-Hanssen, E. 1990, ApJ, 356, 720

Schmieder, B., Fang, C., \& Harra-Murnion, L. K. 1998, Sol. Phys., 182,447

Švestka, Z. 1976, Solar Flares (Dordrecht: D. Reidel)

Wülser, J. P., Canfield, R. C., Acton, L. W., et al. 1994, ApJ, 424, 459 